\title{
Advanced cervical leiomyosarcoma with severe comorbidities: a clinical conundrum for optimal management
}

\author{
Pankhuri Dubey*, Sandhya Gadre
}

Department of Obstetrics and Gynecology, Chirayu Medical College and Hospital, Bhopal, Madhya Pradesh, India

Received: 01 July 2020

Accepted: 06 July 2020

\section{*Correspondence:}

Dr. Pankhuri Dubey,

E-mail: pankhuridubey@gmail.com

Copyright: (C) the author(s), publisher and licensee Medip Academy. This is an open-access article distributed under the terms of the Creative Commons Attribution Non-Commercial License, which permits unrestricted non-commercial use, distribution, and reproduction in any medium, provided the original work is properly cited.

\begin{abstract}
Leiomyosarcoma of uterine cervix constitute a very rare but aggressive group of neoplasms of the cervix with poor prognosis. Although recognised as a distinct entity, treatment algorithms and overall management strategies rely on suggestions from uterine counterparts as robust data on management of the cervical leiomyosarcoma, especially when advanced, is scarce. Present case was a 51-year-old perimenopausal woman with acyclic vaginal bleeding and dyspnoea on ordinary physical activity. On examining, a firm hypogastric mass of 14 weeks was found abdominally and a pedunculated, polypoidal hard mass of $5 X 5 \mathrm{~cm}$ with a $2 \mathrm{~cm}$ thick peduncle coming out of endocervical canal (confirmed on CT) was noted hanging outside vagina. Histopathology suggested cervical leiomyosarcoma. Triple vessel disease and severe cardiac dysfunction $(\mathrm{LVEF}=30 \%)$ with large left ventricular thrombus rendered the patient at very high risk for mortality during surgery. Systematic comorbidity assessment with due consideration to riskbenefit ratio of all treatment options was undertaken and neoadjuvant chemotherapy was started after multidisciplinary recommendation. Patient tolerated the first cycle of chemotherapy well but died of a sudden cardiac arrest after one week. Advanced age and stage are poor predictors for survival in patients with aggressive cervical leiomyosarcomas, even more so, in those with severe comorbidities. Meticulous clinical evaluation and systematic incorporation of comorbidity indices in decision-making for tailored cancer treatment is imperative in arriving at a justified plan of action for this rare and aggressive entity.
\end{abstract}

Keywords: Cervical, Charlson-comorbidity index, Comorbidities, Leiomyosarcoma

\section{INTRODUCTION}

Cervical leiomyosarcomas are distinctly rare and aggressive tumours with poor prognosis. Grouped under Sarcomas, an uncommon group constituting less than $1 \%$ of the all cervical malignancies, Leiomyosarcoma of the cervix represent a very small fraction of cervical tumours $(0.21 \%$ of all cervical malignancies $) .{ }^{1}$ Due to extreme rarity, definite management guidelines are scarce and adopting a systematic treatment algorithm is difficult. Current accepted standards of treatment derive clues from their uterine counterparts and include integration of surgery, radiation and chemotherapy as deemed fit from case to case. ${ }^{2}$
Authors document a rare case of pedunculated cervical leiomyosarcoma diagnosed preoperatively in a patient with severe cardiac dysfunction. Challenges in holistic management of a patient with rare aggressive cancer with severe comorbidities are discussed.

\section{CASE REPORT}

A 51-year-old perimenopausal woman presented with acyclic vaginal bleeding with mass coming out per vaginum for 6 months and dyspnea on ordinary physical activity for 1 year. On examination the patient had severe pallor and abdominally, a firm hypogastric mass corresponding to 14 weeks of gravid uterus was felt. 
Local and per vaginal examination revealed a mass $(5 \times 5$ $\mathrm{cm})$ with irregular surface and greyish pink appearance hanging outside vagina. The mass was hard, pedunculated and a thick stalk of $2 \mathrm{~cm}$ thickness could be felt arising from the endocervix (Figure 1).

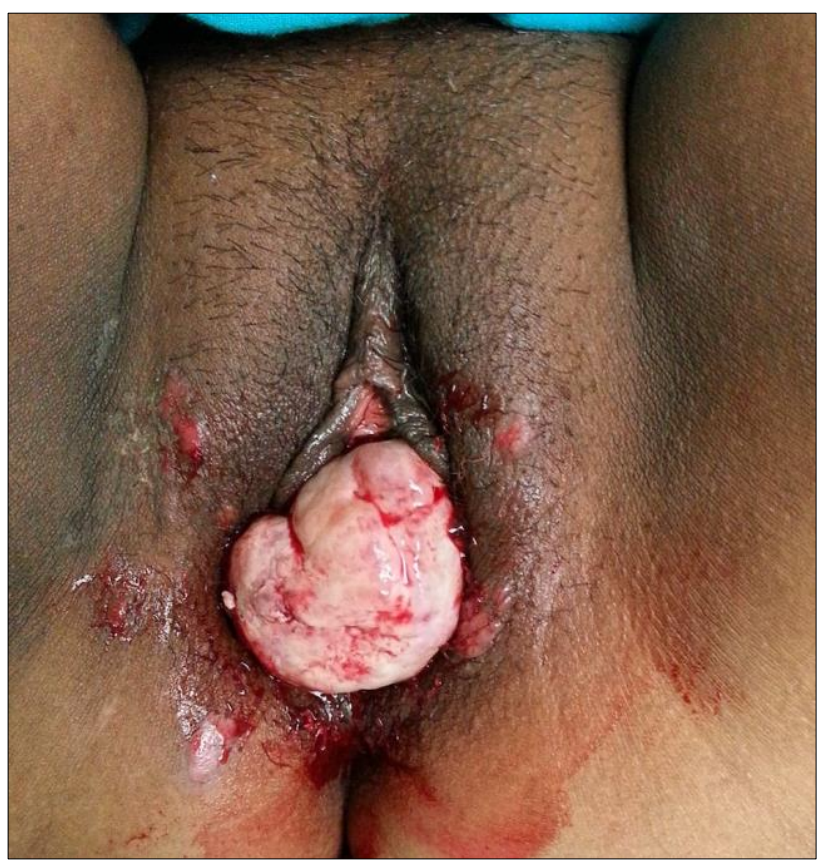

Figure 1: Clinical presentation of the cervical leiomyosarcoma. Mass was noted outside vagina and a thick stalk could be traced to endocervix.

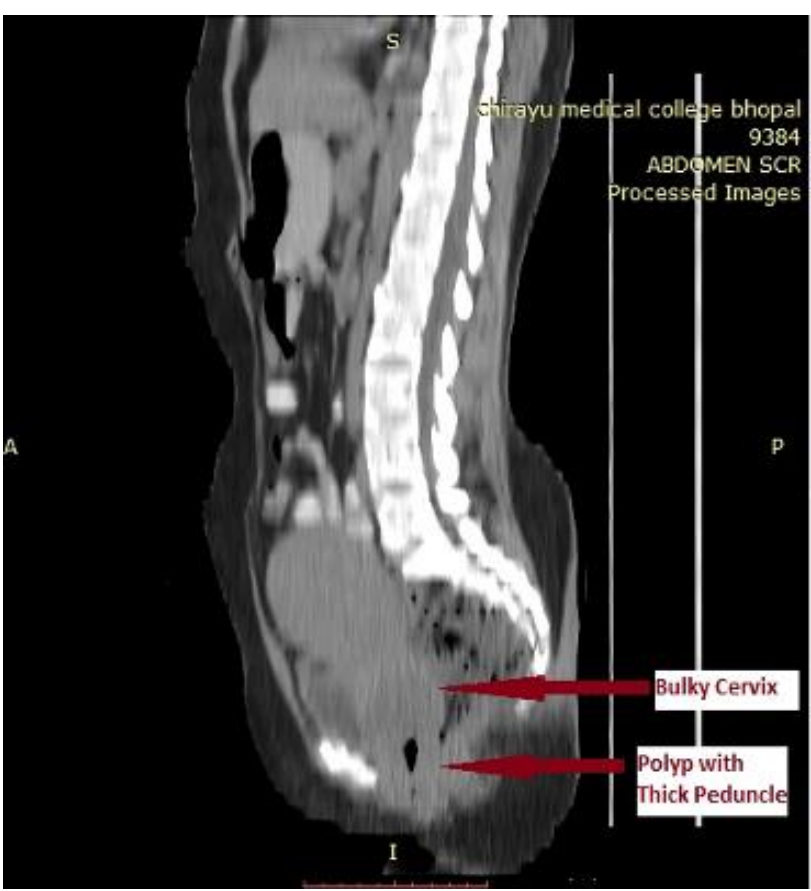

Figure 2: CT scan of bulky cervix with large irregularly marginated polypoidal mass in endocervical canal. Multiple enlarged mesenteric, retro peritoneal and bilateral iliac lymph nodes were also noted.

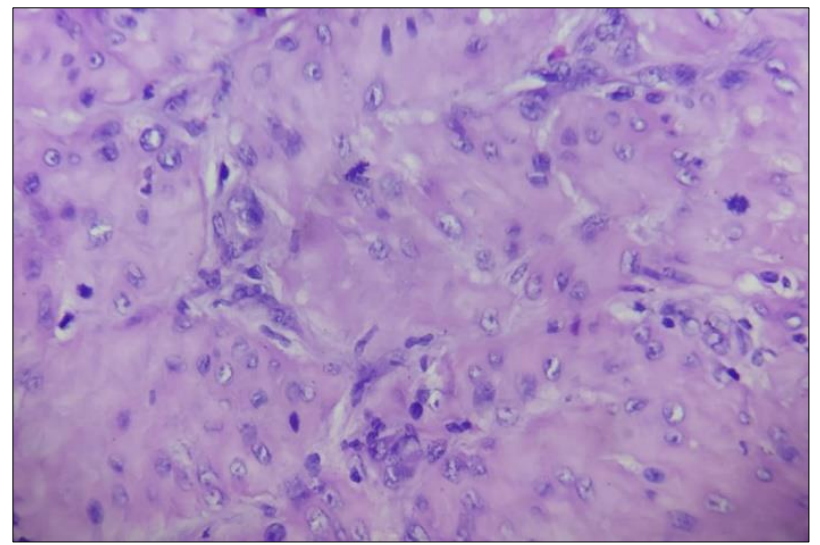

Figure 3: Haematoxylin and eosin stain section of pleomorphic spindle cells with brisk mitosis (Mitosis averaging 9-11/10 HPF).

Parametrium was indurated. Haemoglobin at presentation was $6 \mathrm{gm} \%$. Rest of the biochemical parameters and chest X-ray were normal. She was a known hypertensive and cardiac evaluation revealed triple vessel coronary artery disease, severely reduced LVEF (30\%) and a large LV apical thrombus $(1.5 \times 1.5 \mathrm{~cm})$. Imaging (CT scan) revealed bulky cervix with a hypoechoic, well defined but irregularly marginated polypoidal mass in the endocervical canal (Figure 2). Multiple enlarged mesenteric, retro peritoneal and bilateral iliac lymph nodes were also noted. Biopsy from the polypoidal mass showed pleomorphic spindle cells with brisk mitosis (Mitosis averaging 9-11/10 HPF) suggestive of cervical leiomyosarcoma (Figure 3). The patient was planned for radical hysterectomy, however severe cardiac dysfunction rendered the case operable under very high risk (ASA grade IV), to which the patient and her family did not consent. Multispecialty evaluation of case was done with involvement of cardiologists, onco-surgeons, radiation and medical oncologists and decision to initiate neoadjuvant chemotherapy (doxorubicin and ifosfamide) was taken after informed consent. Patient tolerated the first cycle of chemotherapy well but died of sudden cardiac arrest in the follow up period.

\section{DISCUSSION}

Uterine leiomyosarcomas are known for being elusive to preoperative detection and are often diagnosed incidentally in post-operative specimens on histopathology. On the contrary, the rare leiomyosarcomas of cervix, owing to their location often grant an opportunity to biopsy and diagnose them preoperatively, as in this case.

Primary cervical sarcomas, of which leiomyosarcoma cervix constitute a very small fraction, are generally known to have very poor prognosis due to highly aggressive nature. Patients are $60 \%$ more likely to die of sarcomas compared to those with squamous cell carcinoma, warranting aggressive approach in 
management of these tumours. ${ }^{3}$ Planning treatment, especially for cervical leiomyosarcoma, is however not easy. WHO classification of tumours of female reproductive organs identifies leiomyosarcoma of cervix as an entity distinct from uterine leiomyosarcoma, yet no separate standardized guidelines for management of cervical leiomyosarcoma exist. ${ }^{4}$ Management strategies are therefore conjectured based on the protocols for management of leiomyosarcoma uteri. This consists of multimodality treatment including radical surgery, adjuvant radiation, and chemotherapy with docetaxel, gemcitabine, doxorubicin, and ifosfamide. ${ }^{5}$ Even so, definitive treatment algorithms are lacking and various reported approaches show wide variation.

FIGO cancer report also does not recognize a separate staging system for cervical leiomyosarcoma, therefore staging relies on FIGO Staging for uterine sarcomas which, when utilized, classified the patient as stage III C since pelvic lymph nodes were involved. ${ }^{6}$ Planning treatment is even more challenging in patients presenting with advanced stage and severe comorbidities. This case patient was deemed very high risk for surgery (American society of anaesthesiologists' score for physical status or ASA grade IV) due to severe cardiac dysfunction. Possible treatment strategies included radical hysterectomy under very high risk followed by radiation and chemotherapy, or CABG followed by surgery with adjuvant chemo-radiation. Excision to debulk the pedunculated mass similar to polypectomy as described in one study was also considered, however patient did not consent for any surgical therapy owing to high risk of mortality. ${ }^{7}$ Chemotherapy with recommended agents carried inherent risk of cardiotoxicity however multidisciplinary decision of initiating chemotherapy was adjudged to be the most acceptable treatment option for the present case. Patient succumbed to sudden cardiac arrest within one week of initiating chemotherapy which was, reasonably, not unexpected. This case demonstrates the challenge in striking an optimal balance to achieve acceptable survival in a patient with rare neoplasm and severe life threating comorbid illnesses.

Comorbidities have an undeniable impact on risk-benefit ratio of many treatment decisions especially in older patients with cancer. Evidence from studies on cancer with comorbidities suggests therapy outcomes in such patients is usually inferior as patients are less likely to receive treatments with curative intent. ${ }^{8}$ Uncertain outcomes essentially appear in patients with comorbid illnesses as common eligibility criteria routinely renders older adults with comorbidities ineligible for clinical trials and heterogenous response of such patients, even with well-established treatment protocols for the more common cancers, is not unusual. Besides fundamental inferior outcomes due to comorbidities, aggressive advanced cancers like leiomyosarcoma cervix as in this case patient add to the burden of poor prognosis manifold. As such, there is an irrefutable need for standardized comorbidity assessment with inclusion of measures of comorbidity like Charlson Comorbidity Index (CCI), Cumulative Illness Rating Scale (CIRS), Index of Coexistent Disease (ICED), and the KaplanFeinstein Index (KFI) in research and patient care. ${ }^{9}$ Till definitive management recommendations are formulated for rare cancers like leiomyosarcoma cervix, these may guide clinicians to optimally strategize available treatment options with active involvement of patients and their families. Charlson comorbidity index employs 19 medical conditions weighted 1 to 6 to predict 10 -year survival in patients with multiple comorbidities. ${ }^{10}$ Though developed and validated in 1987, the index is quite underutilized even today in both research and clinical settings. The tool assists not only in systematic assessment but also in realistic counselling of the patient and family. In this case patient had a Charlson comorbidity index score of 9 which placed her at $0 \%$ rate for survival which was instrumental in shared decision making.

\section{CONCLUSION}

Cervical leiomyosarcoma, and rare cancers in general presenting in patients with severe life-threatening comorbidities exemplify a clinical conundrum, as currently treatment guidelines are uncertain and debatable at best. Furthermore, comorbidities alter the risk and benefit of cancer treatment and thus should be systematically analysed with early multidisciplinary involvement for establishing a rational treatment plan.

\section{Funding: No funding sources \\ Conflict of interest: None declared \\ Ethical approval: Not Required}

\section{REFERENCES}

1. Dhull AK, Adarsh C, Kaushal V, Marwah N. The uncovered story of leiomyosarcoma of the cervix: a rare case report and review of literature. Case Reports. 2013 Mar 20;2013:bcr2013008616.

2. Bhatia V, Taksande R, Natekar A, Ali Z. Cervical leiomyosarcoma: a rare entity. Southern Africa J Gynaecol Oncol. 2015;7(2):64-6.

3. Bansal S, Lewin S, Burke W, Deutsch I, Sun X, Herzog T, et al. Sarcoma of the cervix: Natural history and outcomes. Gynecol Oncol. 2010;118(2):134-8.

4. Kurman RJ, Carcangiu ML, Herrington CS, Young RH. WHO classification of tumours of female reproductive organs. Lyon: International Agency for Research on Cancer. World Health Organization. 2014:135-47.

5. Jayaram VK, Parikshith J, Narayanan GS, Tiwari R, Veena R, Prathima S, et al. Multimodality management of leiomyosarcoma of the cervix. E Cancer Med Sci. 2018:12.

6. Mbatani N, Olawaiye A, Prat J. Uterine sarcomas. Int J Gynecol Obstet. 2018;143:51-8. 
7. Muhammad Aminu B, Makama Dattijo L, Abdullahi $\mathrm{K}$, Chama C. Polypectomy as a complete therapy for leiomyosarcoma of the cervix uteri: an unusual treatment. Int J Women's Health Reprod Sci. 2017;7(3):412-4.

8. Sarfati D, Koczwara B, Jackson C. The impact of comorbidity on cancer and its treatment. A Cancer J Clin. 2016;66(4):337-50.

9. Williams GR., Mackenzie A, Magnuson A, Olin R, Chapman A, Mohile S, et al. Comorbidity in older adults with cancer. J Geriatric Oncol. 2016;7(4):24957.
10. Charlson ME, Pompei P, Ales KL, MacKenzie CR. A new method of classifying prognostic comorbidity in longitudinal studies: development and validation. J Chronic Dis. 1987;40(5):373-83.

Cite this article as: Dubey $\mathrm{P}$, Gadre S. Advanced cervical leiomyosarcoma with severe comorbidities: a clinical conundrum for optimal management. Int $\mathbf{J}$ Reprod Contracept Obstet Gynecol 2020;9:3469-72. 